\title{
Bilateral Simultaneous Cochlear Implantation in Children: Report of a Case and Review of Literature
}

\author{
Ajoy Mathew Varghese $\cdot$ John Mathew \\ Arun Alexander · K. Thenmozhi · G. L. Evangelin • \\ Mary Kurien
}

Received: 18 January 2009/ Accepted: 27 March 2009/Published online: 12 April 2011

(C) Association of Otolaryngologists of India 2011

\begin{abstract}
The benefits of bilateral cochlear implant in adults are well established. Auditory ability in children is also substantially improved by binaural hearing. We report the first case of bilateral simultaneous paediatric cochlear implant in India and discuss the merits of bilateral implant.
\end{abstract}

Keywords Cochlear implant · Paediatric · Bilateral ·

Simultaneous

\section{Introduction}

Bilateral cochlear implantation (CI) is becoming increasingly common in clinical practice. The benefits of bilateral implants in adults have been previously established [1]. The data available in children are limited because of difficulties related to data collection in young children due to their shortened attention span and the complexity of the standardized procedures testing binaural hearing. The limited available data from studies in the paediatric age group suggest that not only is bilateral implantation more effective than unilateral or bimodal stimulation, but improved auditory abilities are seen if the implantations are performed simultaneously [2]. We report the first simultaneous bilateral $\mathrm{CI}$ in the paediatric age group in India.

\section{Case Report}

A 22-month-old baby, born of a second degree consanguineous marriage, was suspected to have hearing loss at

A. M. Varghese $(\bowtie) \cdot$ J. Mathew · A. Alexander .

K. Thenmozhi · G. L. Evangelin · M. Kurien

Christian Medical College, Vellore, India

e-mail: ajoymathew@gmail.com
1 year of age. She was fitted with bilateral hearing aids after confirmation of her deafness. On audiological evaluation, DPOAE was absent and there was no response on BERA even with maximal stimulation. MRI and high resolution CT scan of the temporal bone revealed normal cochlea and vestibulocochlear nerves. After extensive discussion and counseling, bilateral cochlear implantation was done in the same anaesthetic sitting. Bilateral cortical mastoidectomy was done through postaural approach and cochleostomy done through a posterior tympanotomy. Bilateral HiRes $90 \mathrm{~K}$ with Hi Focus Helix electrodes (Advanced Bionics) were implanted. The implants were switched on after 4 weeks with mapping done on subsequent days. Both sides were synchronized with ease and the child had remarkable speech scores after auditory verbal therapy. Standard listening skills profile improved from 0 to 30 in 3 months.

\section{Discussion}

The management of profound hearing impairment has been transformed with the advent of cochlear implantation during the 1980's, and the outcomes have continued to improve over time. Although unilateral implants in patients with bilateral profound hearing loss (HL) provide substantial benefits, normal hearing is not restored, even in the implanted ear. Recipients still experience significant auditory handicap because of this residual defect in combination with their severe or profound HL in the contralateral ear. This handicap is most keenly experienced in multi-talker situations, the so-called cocktail party phenomenon [2].

The advantages of bilateral hearing are well known. Specifically, research has demonstrated that bilateral hearing allows for improved detection of low-level sounds, 
improved localization, improved speech recognition in noise, and optimal access for sounds originating from either side of the head [1]. Bilateral implantation also ensures that the 'better ear' is always implanted. Moreover, in the event of device failure or local infection around the components, the second implant will offer valuable insurance against an unwelcome return to profound HL [2]. Bichey and Miyamoto, in a prospective study, found an improvement in the quality of life and a favourable costutility associated with bilateral $\mathrm{CI}$ in patients with profound hearing loss [3]. Bilateral paediatric CI is becoming common in clinical practice. Benefit appears to be gained if a second implant is provided after a short interval and in as young an age group as possible [2, 4]. Based on research published regarding the plasticity and maturation of the auditory system in mammals and the presence of sensitive or critical periods for hearing and speech development, it is plausible to assume the presence of critical periods for the development of binaural hearing and the optimal bilateral integration of both ears [5]. Studies in animal models have shown that spiral ganglion survival is decreased in the nonstimulated ear and intracochlear electrical stimulation promotes survival of spiral ganglion neurons. The normal cochleotopic organization of the inferior colliculus is severely reduced if electrical stimulation is not initiated at an early age. Observations in rehabilitation indicate that the age at implantation and the time lag between implants affect the intensity of training needs and might influence the synchronization of both auditory impressions [6].

The benefits of early restoration of binaural hearing far outweigh the professed risks of bilateral CI. One of the concerns about doing a bilateral $\mathrm{CI}$ is that one ear cannot be "saved" for probable technical or medical innovations in the future. However, it has been found that the P1 cortical auditory-evoked response latency (generated by auditory thalamic and cortical sources) from the second implanted ear in sequentially implanted children was less delayed at hook-up than the P1 latency from their first implanted ear [1]. Moreover, in patients who received simultaneous early bilateral CI, the P1 latency was near normal limits by 1 month after implantation. It is likely that simultaneous early implantation creates an environment in which both the crossed and the uncrossed fibres of the central auditory system on a given side are being simultaneously stimulated. Bauer et al. theorize that synergistic (ipsilateral and contralateral) stimulation facilitates rapid development of central auditory pathways. Therefore, "saving" one ear for probable innovations in the future would actually result in depriving the prelingually deafened child from the benefits of binaural hearing.

Another concern that has been voiced is that bilateral simultaneous CI involves prolonged surgery and consequently, increased risks of anaesthesia and surgical complications. However, it has been found that children tolerate bilateral simultaneous surgery well and no long term vestibular complications have been reported to date. Several workers have found that even in prolonged surgery, the duration of surgery is not a risk factor for increased anaesthesia-related complications. On the other hand, the age of the patient and the experience of the anaesthesiologist were found to be risk factors. Therefore, prolonged surgery may not independently be a risk factor for paediatric CI [7].

Simultaneous surgery allows a more seamless adjustment to the two devices, particularly in young children. Normal cochlear anatomy, no other neurological or medical comorbidities and strong conscientious parental involvement are important prerequisites for bilateral implant. Children between 12 and 36 months with profound HL and no notable aided benefit should be considered for simultaneous bilateral implant.

\section{Conclusion}

The available evidence supports the current trend towards bilateral CI. None of the studies have found a disadvantage in using a second implant. On the contrary, all the studies have demonstrated, to varying degrees, that the auditory ability investigated was substantially improved by binaural hearing. Hence, even in India, in spite of financial constraints, bilateral CI is an option to be considered while discussing with the parents.

\section{References}

1. Bauer PW, Sharma A, Martin K, Dorman M (2006) Central auditory development in children with bilateral cochlear implants. Arch Otolaryngol Head Neck Surg 132:1133-1136

2. Murphy J, O’Donoghue G (2007) Bilateral cochlear implantation: an evidence-based medicine evaluation. Laryngoscope 117:1412-1418

3. Bichey BG, Miyamoto RT (2008) Outcomes in bilateral cochlear implantation. Otolaryngol Head Neck Surg 138:655-661

4. Peters BR, Litovsky R, Parkinson A, Lake J (2007) Importance of age and postimplantation experience of speech perception measures in children with sequential bilateral cochlear implants. Otol Neurotol 28:649-657

5. Kral A, Hartmann R, Tillein J, Heid S, Klinke R (2002) Delayed maturation and sensitive periods in the auditory cortex. Audiol Neurootol 6:346-362

6. Kuhn-Inacker H, Shehata-Dieler W, Muller J, Helms J (2004) Bilateral cochlear implants: a way to optimize auditory perception abilities? Int J Pediatr Otolaryngol 68:1257-1266

7. Johar M, Ho A, Wagner CS, Linder T (2008) Ear surgery in infants under one year of age: its risks and implications for cochlear implant surgery. Otol Neurotol 29:310-313 